\title{
A comparative study of twill weave reinforced composites under tension-tension fatigue loading: Experiments and meso-modelling
}

\author{
J. Xu ${ }^{\text {a,* }}$, S.V. Lomov ${ }^{\text {a }}$, I. Verpoest ${ }^{\mathrm{a}}$, S. Daggumati ${ }^{\mathrm{b}}$, W. Van Paepegem ${ }^{\mathrm{b}}$, J. Degrieck ${ }^{\mathrm{b}}$ \\ ${ }^{a}$ KU Leuven, Department of Materials Engineering, Kasteelpark Arenberg 44, B-3001 Leuven, Belgium \\ ${ }^{\mathrm{b}}$ Ghent University, Dept. of Materials Science and Engineering, Technologiepark-Zwijnaarde 903, 9052 Zwijnaarde, Belgium
}

\section{A R T I C L E I N F O}

\section{Article history:}

Available online 30 September 2015

\section{Keywords:}

Meso-scale

Textile composite

Fatigue damage

Finite elements

\begin{abstract}
A B S T R A C T
The tension-tension fatigue characteristics of two types of twill weave carbon/epoxy composite materials have been experimentally investigated. The fatigue strain vs load cycle number is calculated with the help of digital image correlation (DIC). The increase of displacement amplitude and the degradation of the fatigue moduli for these two materials are studied and compared. The fatigue strengths obtained from fatigue tests are used to compare with those predicted by the model and good agreements are obtained. The stress fields from meso-scale FE models are used to analyse the stress concentration that leads to the fibre rupture in the warp tows.
\end{abstract}

(c) 2015 Elsevier Ltd. All rights reserved.

\section{Introduction}

Woven composites are gaining popularity in the industry. However, as it is known, the crimp of the tows decreases the inplane mechanical properties. As concluded in Ref. [1] damage and strength of the textile composite under axial loads are strongly affected by the fibre crimp. On the one hand, high flexure introduces severe local stress concentration, especially at the tow crossover locations. On the other hand, the shear-bending interaction between warp and weft tows leads to an early inter-fibre matrix cracking in the tows transverse to the loads.

Many factors affect the mechanical behaviour of the woven composites. These factors include: (1) the mechanical properties of the fibre and matrix material, (2) the fibre/matrix interface, (3) the fibre volume fraction (VF) of the reinforcement materials, (4) the processing techniques (resin transfer moulding vs autoclave) and the processing parameters (curing time, temperature, pressure...), and (5) the reinforcement's geometrical parameters such as tow width, tow thickness, unit cell size and crimp.

Detailed literature reviews on fatigue analysis of textile composites have been provided by Degrieck and Van Paepegem [2], Post [3], Passipoularidis and Brøndsted [4] and Xu [5], respectively. According to $\mathrm{Xu}$ [5], the existing fatigue models can be classified into three categories: Miner's-rule-like models, phenomenological models and progressive damage models. In the recent, intensive experimental investigations have been carried out on fatigue

\footnotetext{
* Corresponding author.

E-mail address: xujian1028@hotmail.com (J. Xu).
}

behaviour of woven composites [6,7], and comparative studies were conducted by Carvelli et al. [8,9]. In Ref. [8] the non-crimp stitched UD and non-stitched UD are experimentally studied and the stitching effects are analysed. The on-axis fatigue resistance is enhanced by the structural stitching but weakened in the orthogonal direction. In Ref. [9], the 3D non-crimp orthogonal woven composite and 2D plain weave laminate are comparatively investigated. The conclusion is that the former composite has a longer fatigue lifetime and later damage onset. Nishikawa et al. [10] compared newly developed plain weave spread tow (cross-section aspect ratio $=20 \mathrm{~mm}: 0.05 \mathrm{~mm}=400$ ) carbon/epoxy to conventional plain woven composites through static and fatigue tests. The spread tow composite showed an increase of static strength about $14 \%$ and 15 times prolongation of the fatigue life. A more comprehensive collection of the studies on textile composites' fatigue behaviour has been recently published [11] that covers the experimental measurement, observation, numerical prediction and industrial application.

In this paper two types of twill weave carbon fibre epoxy composites are studied: (i) carbon/epoxy twill weave $3 \mathrm{~K}$ tow laminates (CET3K) with (tow) width: thickness $=2 \mathrm{~mm}: 0.11 \mathrm{~mm}=18$, and (ii) carbon/epoxy twill weave $12 \mathrm{~K}$ tow laminates (CET12K) with (tow) width: thickness $=5.45 \mathrm{~mm}: 0.16 \mathrm{~mm}=34$. The tow crimps of these two impregnated fabrics are $0.85 \%$ and $0.28 \%$, respectively. These two selected materials have the same fibre/resin system and fibre volume fraction, and are processed using autoclave following the same curing cycle, in order to avoid influences of the aforementioned factors (1)-(4). In author's earlier work [1], these two materials were experimentally and numerically characterised 
under static loadings. With higher crimp the CET3K exhibits earlier damage initiation in weft tows and remarkably lower strength than that of CET12K.

In the current work, the materials' responses to fatigue loadings are investigated through tension-tension fatigue tests and the recently proposed fatigue model [12]. The goal of the experiments is to acquire the modulus-life curves and $S-N$ plots. The test results of these two materials are compared and the different behaviour is analysed. Meantime, the meso-FE model (Fig. 8) is used to quantitatively demonstrate stress concentrations that lead to fibre breakage and final failure, and differences in stress-strain fields for the two materials.

The impregnated tows are represented in the model as unidirectional composite (UD). In order to produce the $S-N$ curves to feed fatigue model, UD samples made from M10.1/T700S prepreg system, the same as in CET3K (VF $=55.2 \pm 0.08 \%$ ) and CET12K $(\mathrm{VF}=53.6 \pm 0.17 \%)$, are tested under tension-tension fatigue loadings, too. The M10.1 resin system that has high fatigue resistance is suitable for low pressure moulding processes allowing a range of processing temperature from $85^{\circ} \mathrm{C}$ up to $150^{\circ} \mathrm{C}$.

\section{Tension-tension fatigue test}

\subsection{Experimental setup}

The fatigue tests are conducted using MTS ${ }^{\circledR} 810$ servo hydraulic testing machine equipped with a fatigue load cell of $100 \mathrm{KN}$ and MTS $^{\circledR} 647$ Side-Loading hydraulic wedge grips. The operation system is TestStar-790.00 digital controller. A sinusoidal-wave constant-amplitude tension-tension fatigue load with stress ratio $R=0.1$ is applied to the samples. The tests are carried out in a conditioned room with a temperature of $18^{\circ} \mathrm{C} .3 \mathrm{~Hz}$ load frequency is uniformly applied to all tests. Two factors have to be considered when defining the load frequency:

(A) The consistency of frequency for all samples: The frequencies of the fatigue loading applied to UD samples ( $S-N$ curves to feed the model [12]) and textile composites are identical. In an ideal case the frequency has little effect on fatigue life of the composites when the temperature is strictly controlled [13].

(B) High level fatigue loadings: As high level fatigue loadings (75-85\% of the static strengths - Table 1) will be applied, the load frequency should be less or equal than $3 \mathrm{~Hz}$ in order to prevent the failure near to the end-tabs. De Baere et al. [13] often observed in-tab failures under fatigue loadings $75 \%$ of the strength at $5 \mathrm{~Hz}$ frequency. He assumed higher frequency leads to higher heat generation between tab and sample surface due to friction which decays the adhesive and hence induces the in-tab failure. Lower than $3 \mathrm{~Hz}$ frequency would not be affordable in fatigue since the total test campaign would be extended to more than one year [5].

\subsection{Acquisition of the fatigue strain}

All the fatigue tests in this work are load-controlled. However, the recorded grip displacement by the machine is not the true elongation of the sample but the summation of the sample elonga-

Table 1

Stress levels (\%) and corresponding fatigue loadings (MPa).

\begin{tabular}{llc}
\hline & CET3K & CET12K \\
\hline Static strength [1] & 960 & 1132 \\
$75 \%$ & 720 & 849 \\
$80 \%$ & 768 & 906 \\
$85 \%$ & 816 & 965 \\
\hline
\end{tabular}

tion and the compliance of the test machine. Use of a dynamic extensometer was not possible because it tended to be detached from the specimen in the very early test stage. Hence a relation between the grip displacement and the true strain in the middle of the sample was measured using optical extensometer (digital image correlation or DIC - Vic $2 \mathrm{D}^{\circledR}$ ) and then this relation applied in fatigue testing to estimate the sample strain. The DIC averaging area $(80 \mathrm{~mm} \times 25 \mathrm{~mm})$ was set to the centre of the sample. The full configuration was explained in Ref. [1].

In the first cycle a very low frequency $(0.01 \mathrm{~Hz})$ tensile load is applied to sample, stretching the sample up to the maximum fatigue load. The longitudinal strain, $\varepsilon_{\text {sample, }}$ and hence the sample's elongation, $D_{\text {sample }}(F)$ can be obtained by Eq. (1):

$D_{\text {sample }}(F)=\epsilon_{\text {sample }} \cdot L_{\text {sample }}$

where $L_{\text {sample }}$ is the sample free length. The total elongation, $D_{\text {total }}(F)$, of the sample and machine is known from the MTS data file. Hence the machine compliance, $D_{\text {machine }}(F)$ vs load $F$ can be obtained as:

$D_{\text {machine }}(F)=D_{\text {total }}(F)-D_{\text {sample }}(F)$,

as sketched in Fig. 1.

After removing the DIC, in the rest of the load cycles, the sample's elongation can be calibrated by Eq. (3):

$D_{\text {sample }}(F)=D_{\text {total }}(F)-D_{\text {machine }}(F)$

where $D_{\text {machine }}(F)$ is the machine tension-deformation curve obtained from Eq. (2). The assumption is taken that the dynamic effect (from $0.01 \mathrm{~Hz}$ to $3 \mathrm{~Hz}$ ) will not give a relevant influence. Sample-grip slippage has to be strictly prohibited during all tests. Therefore a red mark was drawn on the grips aligning to the endtabs to indicate any slippage.

\subsection{Experimental data of carbon/epoxy UD}

The M10.1/T700S prepreg system is processed into UD composite plates by using autoclave as explained in detail in Ref. [5]. The test coupons are prepared following the description in Ref. [1]. The width of the samples is $15 \mathrm{~mm}$ according to ASTM D3039 for UD composites. The mechanical properties are acquired through tensile tests using Instron ${ }^{\circledR} 4505$ at a cross-head speed of $1 \mathrm{~mm} / \mathrm{min}$ combined with extensometer. The mechanical properties in the fibre direction are listed in Table 2.

The fatigue tests have been performed in the fibre direction. Four loading levels are predefined: $70 \%, 75 \%, 80 \%$ and $85 \%$ of the static strength. For each loading level, at least three valid test results are necessary. Fig. 2(a) exhibits the normalised fatigue life

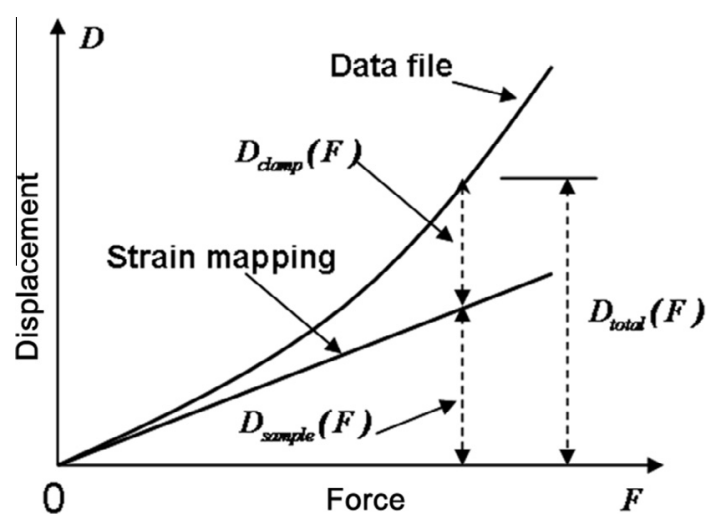

Fig. 1. Sketch showing how to calculate the machine compliance $D_{\text {machine }}(F)$ vs tensile loading by using DIC: the quasi-straight curve is obtained using DIC and the non-linear one is based on the force-displacement data recorded by MTS ${ }^{\circledR} 810$. 
Table 2

Mechanical properties of UD composite through 3 valid test samples in fibre direction.

\begin{tabular}{lll}
\hline $\mathrm{VF}(\%)$ & Strength $(\mathrm{MPa})$ & Modulus (GPa) \\
\hline $55.3 \pm 2.2$ & $1345.1 \pm 77.6$ & $140.3 \pm 7.2$ \\
\hline
\end{tabular}

scatter. The Semi-Logarithmic-Bilinear regression model [14], as discussed in Ref. [12], is used to generate the $S-N$ curve.

The Semi-Logarithmic-Bilinear model in Fig. 2(a) can be represented by the following equations:

$$
\begin{cases}f(N)=1.0 & \left(\log _{10} N \leq 3.15\right) \\ f(N)=-0.09 \cdot \log _{10} N+1.28 & \left(3.15<\log _{10} N \leq 6.47\right) \\ f(N)=0.70 & \left(\log _{10} N>6.47\right)\end{cases}
$$

In Fig. 2(a), the semi-logarithmic curve depicts that the fatigue strength starts decreasing after one thousand cycles and reaches the fatigue limit at about three million cycles. The material reaches fatigue limit ( 3 million cycles) at the load level about $70 \%$ of the static strength.

The on-axis catastrophic failure and the fatigue life are dominated by the fibre bundle rupture or on-axis $S-N$ curve of UD in terms of fatigue modelling. The $S-N$ curve of UD composite in the fibre direction will be used as the input data for the fatigue model to predict the on-axis fatigue life of the CET3K and CET12K. Further, the fatigue data of transverse fibre tension-tension fatigue, in-plane shear fatigue and out-of-plane shear fatigue from Ref. [15] are utilised as input - Fig. 2(b)-(d). The material used in
Ref. [15] is AS4/3501-6 carbon/epoxy system, which may have different matrix mechanical properties and interface properties compared to T700S/M10.1 system. Ref. [15] provides the most complete test data and hence the best choice for the time being. However, for the fatigue behaviour in off-axis direction, where the matrix and fibre/matrix interface properties dominate the damage progression, test samples using the same resin system as in the model are inevitable. Through FE-model, mild compression effects were observed in the weft yarn along the fibre direction, or perpendicular to the layer plane, due to Poisson's ratio. These compressive stresses are trivial and have, however, minor or none contribution to the material damage. Hence, for the time being, the compressive loads are neglected.

\subsection{Experimental results of twill composites}

\subsubsection{Displacement amplitude increase}

The tension-tension fatigue load is applied in warp direction of the material. In a load-controlled fatigue test, the maximum and minimum of the applied load are constant throughout the test. Due to the (i) permanent deformation that is introduced by thermal stress release and fibre straightening and (ii) modulus degradation, the maximum and minimum of the displacement response keep increasing during the fatigue life - Fig. 3. This phenomenon has been reported by Carvelli et al. [8]. Tested under a series of fatigue loadings that have the same load frequency and load ratio, the maximum displacement (peaks) of the $N$-th cycle can be presented as:

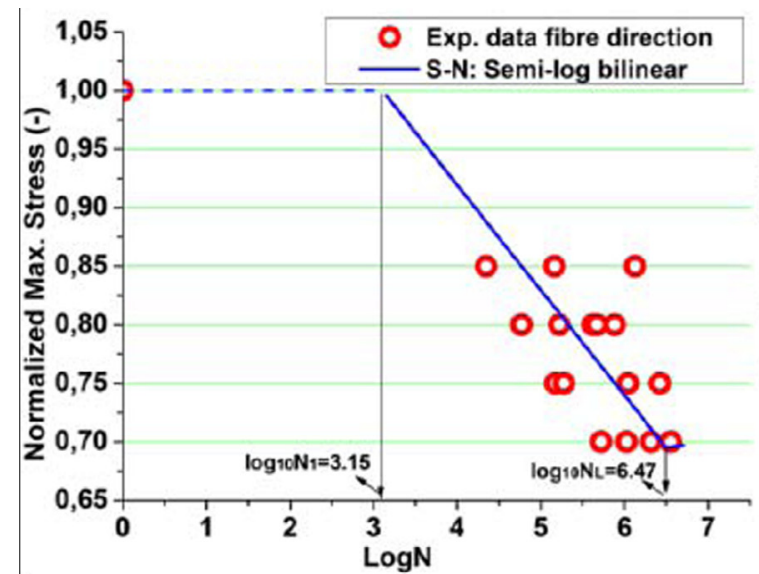

(a)

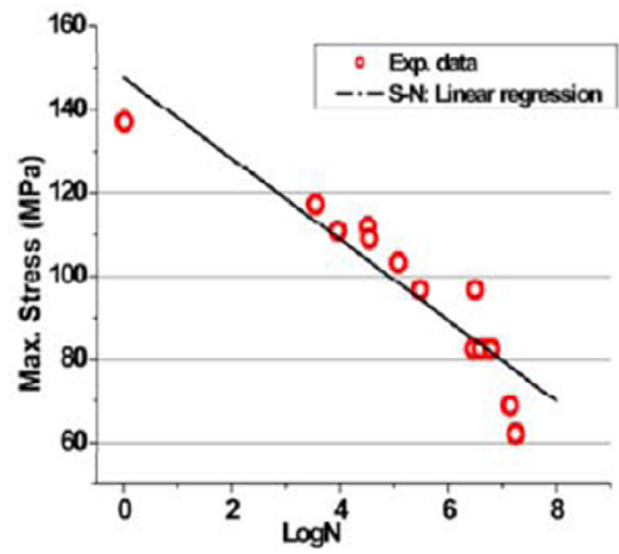

(c)

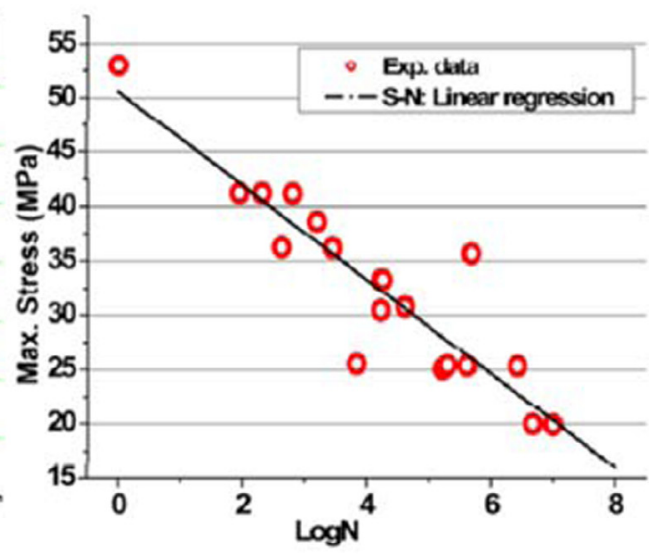

(b)

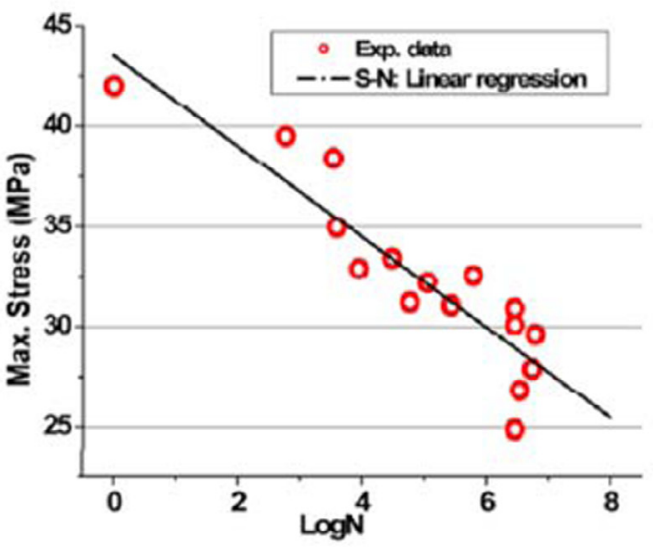

(d)

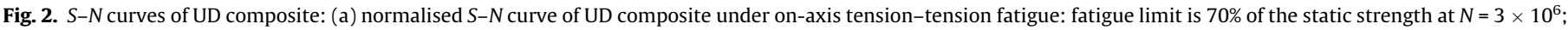
(b) transverse fibre tension-tension; (c) in-plane shear; (d) out-of-plane shear. Data in (b)-(d) are extracted from Ref. [15]. 


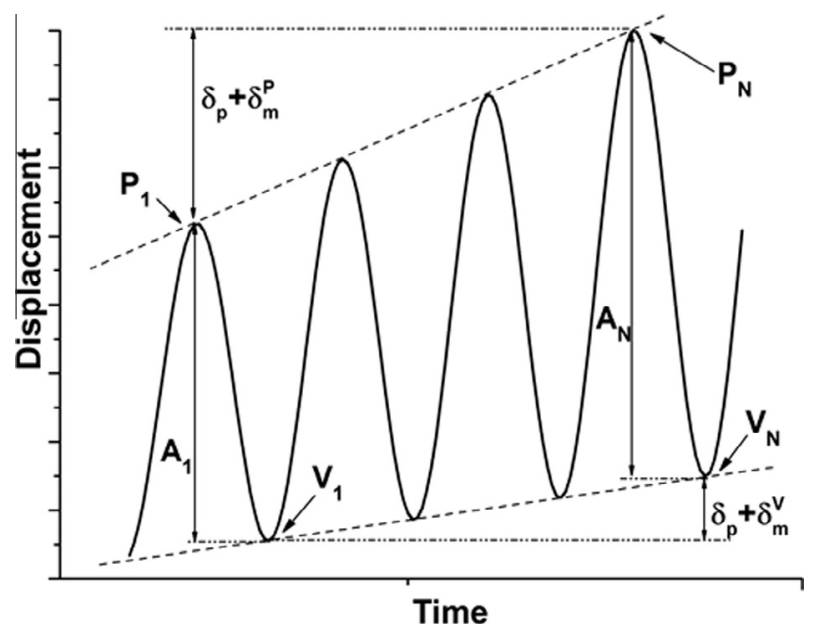

Fig. 3. Sketch for the increase of the displacement peak (maximum), valley (minimum) and amplitude of the sample in fatigue test due to the material degradation and permanent deformation.

$P_{N}=P_{1}+\delta_{p}+\delta_{m}^{P}$

where $P_{1}, \delta_{p}$ and $\delta_{m}^{P}$ are the maximum displacement in the first cycle, the permanent deformation and the deformation due to the reduction of the modulus under maximum load, respectively. Similarly, the minimum displacement (valleys) of the $N$-th cycle can be presented as:

$V_{N}=V_{1}+\delta_{p}+\delta_{m}^{V}$

where $V_{1}$ and $\delta_{m}^{V}$ are the minimum displacement in the first cycle and the additional elongation due to the reduction of the modulus under minimum load. $\delta_{m}^{V}$ is increasing throughout the fatigue life but quasi-constant in an arbitrary load cycle. Subtracting Eq. (6) from Eq. (5), it yields:

$\Delta A=A_{N}-A_{1}=\left(P_{N}-V_{N}\right)-\left(P_{1}-V_{1}\right)=\left(\delta_{m}^{P}-\delta_{m}^{V}\right)$

where $A_{N}, A_{1}$ and $\Delta A$ are displacement amplitude of the $N$ th cycle, displacement amplitude of the first cycle and the increase of the amplitude (AI), respectively. Using Eq. (7) to process the experimental data for CET3K and CET12K, the curves of $\Delta A$ for fatigue tests on different load levels are shown in Fig. 4(a).

In order to show clearly the dependence of moduli's reduction on material type, the test data in Fig. 4 are plotted based on the normalised life cycle number. From Fig. 4(a), it can be observed that: (i) the AI curves are clearly divided into two groups according

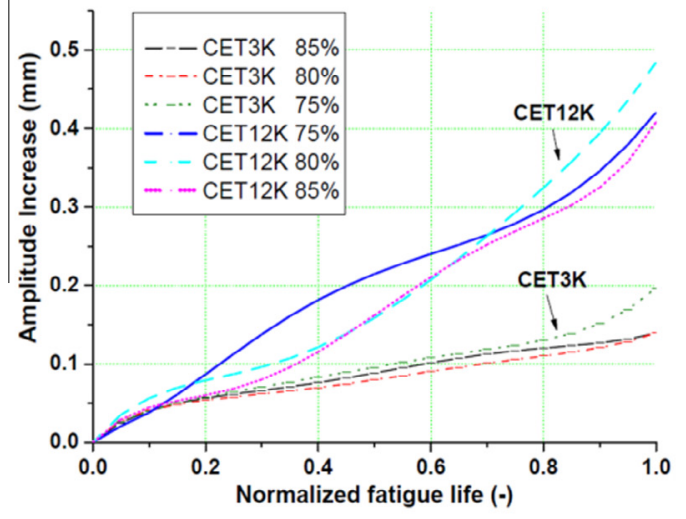

(a) to material types; (ii) for CET12K, the ultimate $\mathrm{AI}$ is in a range between $0.42 \mathrm{~mm}$ and $0.49 \mathrm{~mm}$, higher than that of CET3K, between $0.15 \mathrm{~mm}$ and $0.20 \mathrm{~mm}$; (iii) the ultimate AI tends to be irrelative to the load levels (either $75 \%, 80 \%$ or $85 \%$ of the static strength) but dependent on the material type.

\subsubsection{Degradation of the sample stiffness}

Fig. 4(b) presents the normalised longitudinal fatigue moduli decrease vs normalised fatigue life for CET3K and CET12K on three fatigue load levels. As well the curves are divided into two groups based on material types: CET3K samples have relatively moderate modulus decreases through the tests, $13-17 \%$, compared to those of CET12K, 23-30\%. The higher applied loadings (Table 1) and the higher modulus decreases of CET12K have clearly demonstrated a higher damage tolerance than CET3K, which has been suggested by Fig. 4(a), too. Meantime, the averaged fatigue modulus decrease ( $15 \%$ for CET3K and $25 \%$ for CET12K) will be used in the fatigue model to determine the final fatigue failure as explained in Refs. $[5,12]$.

Sharp drops of the moduli in the first and the last $5 \%$ of the fatigue life are observed. This phenomenon was also reported for cross-ply UD composites in Ref. [16]. The first drop (stage I in Fig. 4(b)) consists of transverse crack formation with increasing density, until a saturation state is reached. In stage II, delamination occurs with gradual breakage of those fibres which have defects. This stage lasts for about $90 \%$ of the fatigue life cycles at a much lower and an approximately linear rate of modulus decrease. In the last drop (stage III), the moduli drop abruptly till the catastrophic failure, formed by massive fibre breakage.

As seen from Fig. 4(b), stage II provides a major contribution (7\% out of total loss $15 \%$ for CET3K and $17 \%$ out of total loss $25 \%$ for CET12K) to the modulus loss in fatigue. Through the morphological study shown in Fig. 5, it is found out that all the CET12K samples, failed at $85 \%, 80 \%$ and $75 \%$, have developed severe fatigue damage accompanied with fibre pull-out and tow splitting. In contrast, CET3K samples kept much better surface finishing. The much flatter reinforcement of CET12K may help the inter-laminar crack propagation and, therefore, form the fatigue damage and delamination. The observed severer fatigue damage of CET12K gives one good reason to the higher displacement AI and higher modulus loss of CET12K in fatigue.

\subsubsection{Normalised modulus vs logarithmic cycle number}

In Fig. 6(a) and (b) the normalised moduli on different load levels for CET3K and CET12K are plotted vs logarithmic life cycles. In these figures, the corresponding three stages of modulus degradation are also marked out for the stress level of $85 \%$. Plotted on

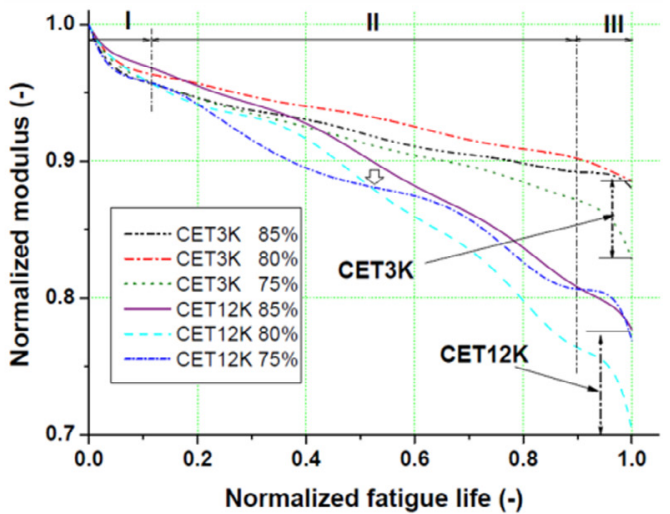

(b)

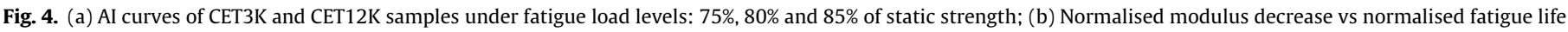
for CET3K and CET12K samples under fatigue loads of $75 \%, 80 \%$ and $85 \%$ of static strength: three damage stages. 


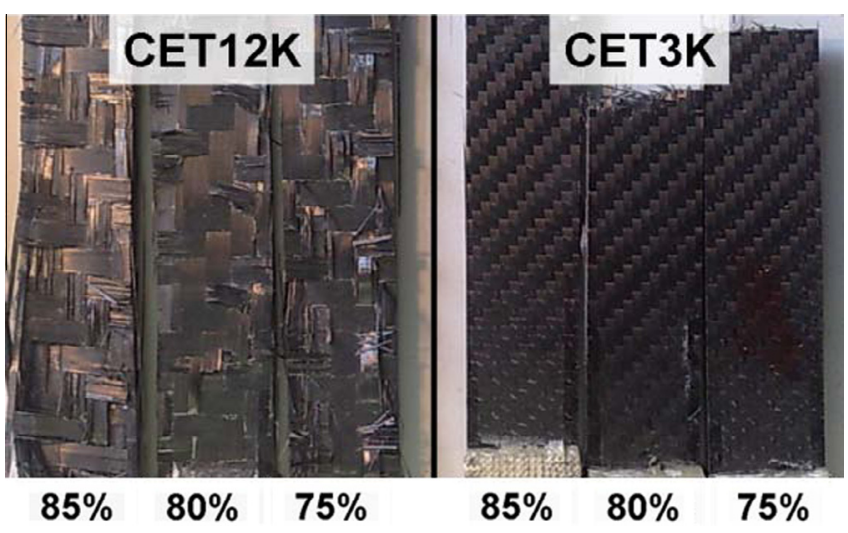

Fig. 5. The morphology of the samples after fatigue failure for CET12K and CET3K. They were fatigued under $85 \%, 80 \%$ and $75 \%$ of strength, respectively. CET $12 \mathrm{~K}$ samples show severe fatigue damage, fibre pull-out and rough surface.

the logarithmic scale of the fatigue life, the moduli curves do not present the three stages as shown in Fig. 4(b). Stage I in Fig. 4(b) is stretched and shows slight decreases (less than $2 \%$ ) before the first 1000 cycles. Right after 1000 cycles (until 10,000 cycles), the moduli start reducing drastically owing to the transverse crack formation that correlates to the first drop in Fig. 4(b). Stage II in Fig. 4 (b) represents gradual degradation of the modulus, showing sharp decrease in the logarithmic plot.

As expected, the degree of the modulus drop depends on load levels: the higher the load is, the faster the modulus reduces.

\subsubsection{S-N plot}

The fatigue strengths of CET3K and CET12K are normalised by their static strengths, $960 \mathrm{MPa}$ and $1132 \mathrm{MPa}$ (Table 1), respectively. Three valid samples were tested for each considered load level. The normalised strengths are plotted in Fig. 7(a) and (b). From these two figures, it is clear that (1) both of CET3K and CET12K reach fatigue limit (one million cycles) at $75 \%$ of the static strength and (2) they have about the same fatigue life under the same normalised fatigue loading. When compared for the absolute values of the maximum fatigue stress in Fig. 7(c), however, the maximum fatigue stress corresponding to the same fatigue life is about $18 \%$ higher for CET12K than that for CET3K, the same ratio as that of static strengths for these two materials.

The crimp is a typical structural feature and also a serious disadvantage for a woven laminate that significantly reduces the strength and introduces highly nonlinear response by high bending torsion loading between crossed tows. Spread tow plain weave laminate [17] with extremely low crimp has been experimentally proven to have superior fatigue tolerance than conventional plain weave laminates. With lower crimp, CET12K (crimp 0.28\%) has proven its greater static and fatigue resistance over CET3K (crimp $0.85 \%)$.

\section{Progressive fatigue damage model}

A meso-FE progressive damage model for textile composites under fatigue loadings has been recently published by authors [12]. This model is used to provide a quantitative study of the fatigue damage as a consequence of stress concentration that is given by tow interlock.

\subsection{Methodology}

This fatigue model is based on the mesoscale (unit cell) FE method - Fig. 8. A unit cell is the minimum repeating unit that possesses the same properties as in the whole material. In term of common textile composites, a unit cell (UC) occupies the same representative tow waviness, tow fibre volume fraction and tow geometrical parameters as in macroscale material. With the periodic boundary conditions (PBCs), the local strain and stress fields in the full-scale material can be reproduced through a meso-FE model. In UC, the impregnated tows can be taken as piecewise UD composites: the $S-N$ curves of UD material will be used as inputs to predict the fatigue characteristics of a textile composite material - Fig. 8. The algorithm will be carried out by repeating step $\mathbf{A}$ and $\mathbf{B}$ :

(A) Quasi-static loading is applied to the unit cell as half cycle: Employing the static damage model [1], the unit cell is loaded up to the magnitude of the maximum fatigue stress $\sigma_{\max }$. Some Gaussian points are identified as failure and their stiffness is degraded following the anisotropic damage model [1]. The degraded properties will be passed to Module $\mathbf{B}$.

(B) Material is 'worn out' due to increasing number of load cycles: The 'N_jump' [18] stands for cycle number that may be a predefined constant value or a number calculated in-situ. No fatigue damage will be evaluated in side this amount of load cycles but only at the end of it. With a predefined ' $N \_j u m p$ ', all the Gaussian points continuously experience the fatigue weakening effect. Governed by the input $S-N$ curves, this weakening effect is the function of the $\mathrm{N}_{\text {jump }}$ and local stresses states. The fatigue weakening

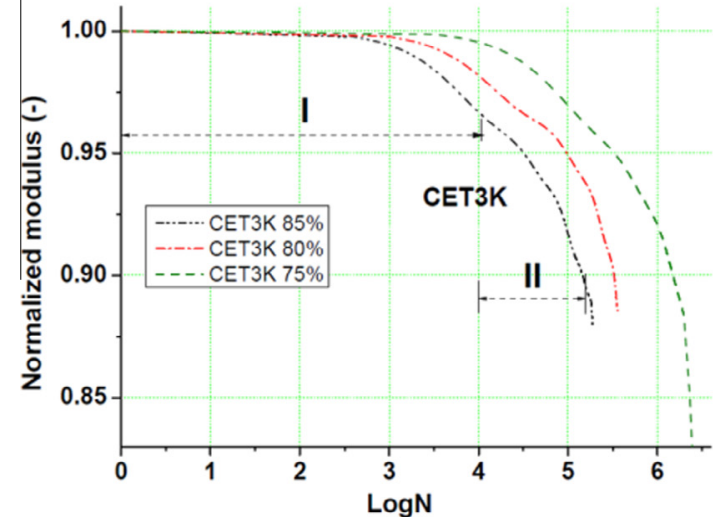

(a)

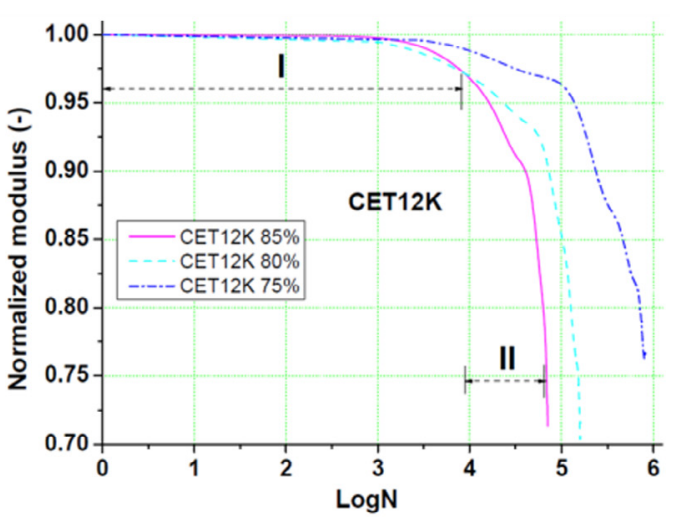

(b)

Fig. 6. Normalised fatigue modulus vs logarithmic load cycle number: (a) CET3K and (b) CET12K. 


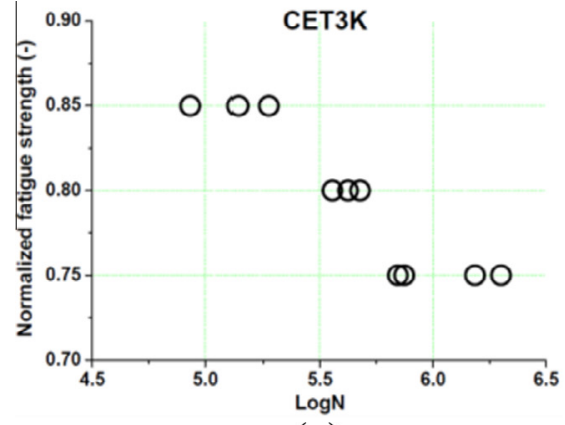

(a)

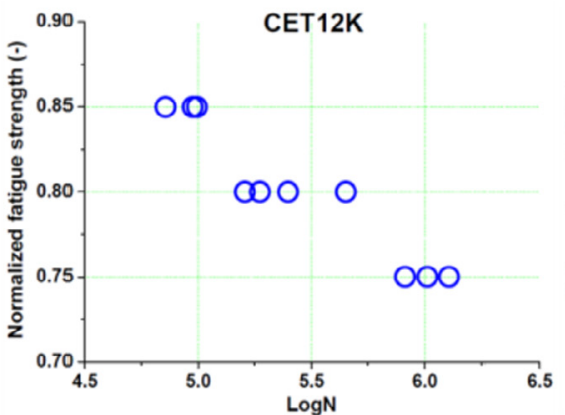

(b)

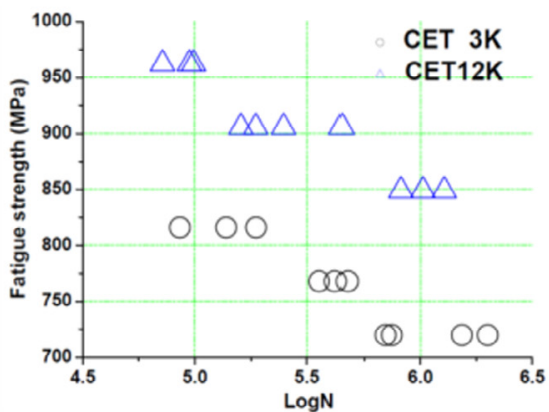

(c)

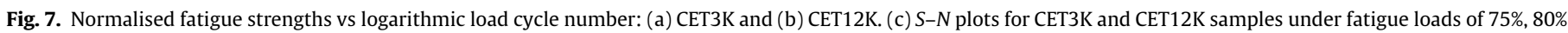
and $85 \%$ of static strengths, respectively.

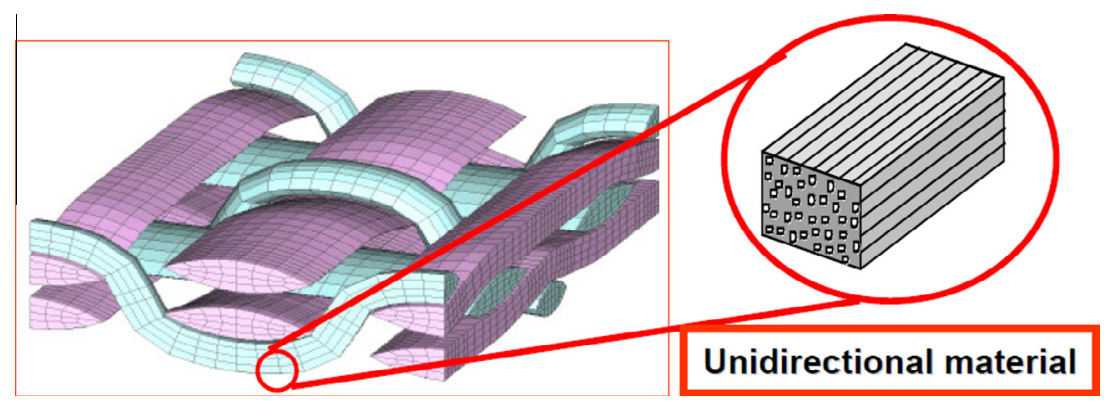

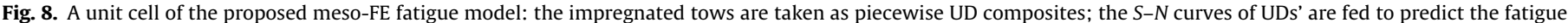
properties of a textile composite material.

effects are cumulated by Palmgren-Miner's rule - Eq. (8). Some Gaussian points are identified as damaged and their stiffness is degraded by the anisotropic damage model; the rest of the Gaussian points keep their stiffness unimpaired. The matrix material is taken as isotropic material, and the Palmgren-Miner's rule and the multi-axial fatigue theory $[19,20]$ - Eq. (9) are applied.

\subsection{Geometrical model}

The mesoscale model of textile composites has to be provided with an adequate geometrical description of a unit cell, which can be created using a textile geometry generator [21,22] - Fig. 9 (a) and converted thereafter to the FE mesh - Fig. 9(b) assisted by a FE mesher $[23,24]$. The FE-mesh will be eventually converted to ABAQUS environment with the appropriate fibre orientation and PBCs - Fig. 9(c).

\subsection{Fatigue failure criteria}

On the one hand, the on-axis fatigue damage should be considered separately because it is governed by breakage of the fibres other than inter-fibre matrix and fibre-matrix interface. On the other hand, the fibre breakage is the primary failure that will degrade all the stiffness components by a factor of 0.01 . During the damage propagation, the local stress amplitudes keep changing due to the stress redistribution introduced by the consecutive stiffness degradation of the neighbouring Gaussian points. In this model, the along-fibre fatigue damage is accumulated using Palmgren-Miner linear damage rule, which yields the residual portion of the fatigue strength of a Gaussian point after a series of block loadings: $\sigma_{1}^{1}, \sigma_{1}^{2}, \ldots, \sigma_{1}^{i}$ with the correspondent cycle number $N_{\text {block }}^{1}, N_{\text {block }}^{2}, \ldots, N_{\text {block }}^{i}$ - Fig. 10.
$R_{1}^{j}=\sum_{k=1}^{j} \frac{N_{\text {block }}^{k}}{N_{1}^{k}\left(\sigma_{1}^{k}\right)}$,

$R s_{1}^{j}=1-R_{1}^{j}$

where $R_{1}^{j}$ is the portion of exhausted strength and $N_{1}^{k}\left(\sigma_{1}^{k}\right)$ is the maximal number of cycle to failure on stress level $\sigma_{1}^{k}$. The fraction $N_{\text {block }}^{k} / N_{1}^{k}\left(\sigma_{1}^{k}\right)$ is called 'damage fraction' for the $k$-th cycle block.

For the matrix-dominated directions, Liu's theory [19], which accounts for multi-axial fatigue and independent to the material type, is applied. Consider a UD, the material coordinate system ' $1-2-3$ ' is defined as such: the coordinate system obeys the right-hand rule; axis 1 follows the fibre direction; axis 2 is parallel to the unit cell plane; axis 3 is perpendicular to the plane $1-0-2$ following the right-hand-rule - Fig. 11.

In the material coordinate system, the 'crack plane' and 'critical plane', based on which Liu came up with his multi-axial fatigue failure criterion, have to be interpreted. The 'crack plane' is a micro-level fracture plane parallel to the fibres, whose normal has an angle $\left(\theta_{\text {crack }}\right)$ with axis 3 - Fig. 11 . In this model, the potential crack plane, a plane experiencing the maximum principal stress, will be numerically sought out. The 'critical plane' can be taken as a coordinate, into which the stresses are transformed and the fatigue damage is evaluated using Eq. (9). Based on the notions of 'crack plane' and 'critical plane', Liu and Mahadevan [19] proposes a criterion for planar stress states - Eq. (9):

$$
\sqrt{\left(\frac{\sigma_{c}}{f(N)}\right)^{2}+\left(\frac{\tau_{c}}{t(N)}\right)^{2}+k\left(\frac{\sigma_{c}^{H}}{f(N)}\right)^{2}}=\beta
$$

This criterion is then extended to composite materials [20]. This model is a second order combination of the peak values (magnitudes) of cyclic normal stress $\sigma_{c}$, shear stress $\tau_{c}$ - and hydrostatic stress $\sigma_{c}^{H}$ (mean normal stress) on the critical plane (subscript ' $c$ ' 


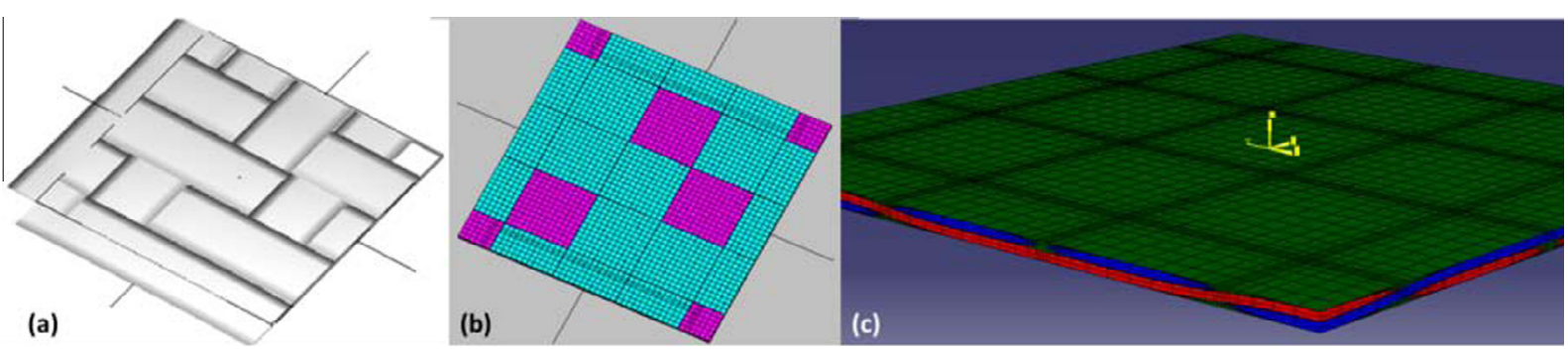

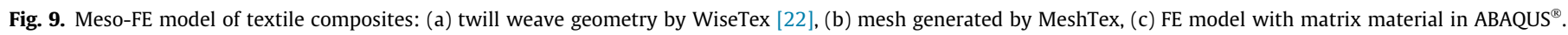

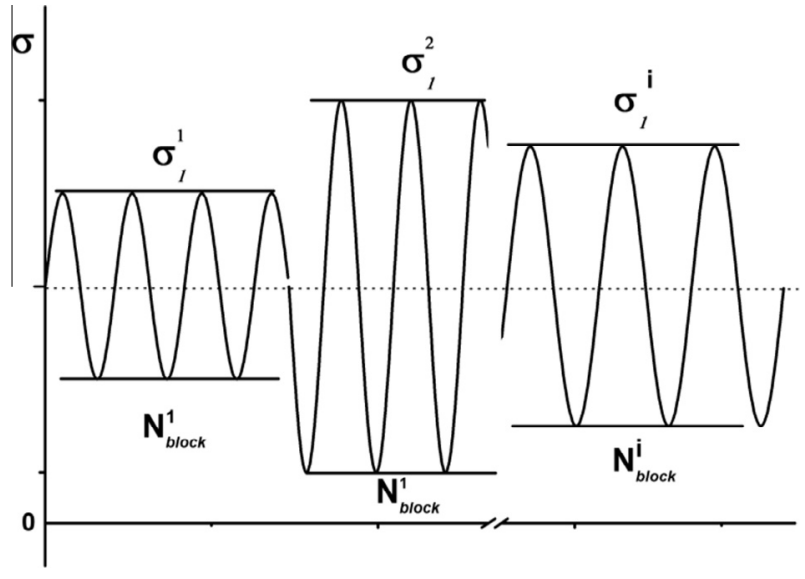

Fig. 10. Application of Palmgren-Miner's linear damage rule to a Gaussian point in fibre direction: constant amplitude loading blocks.

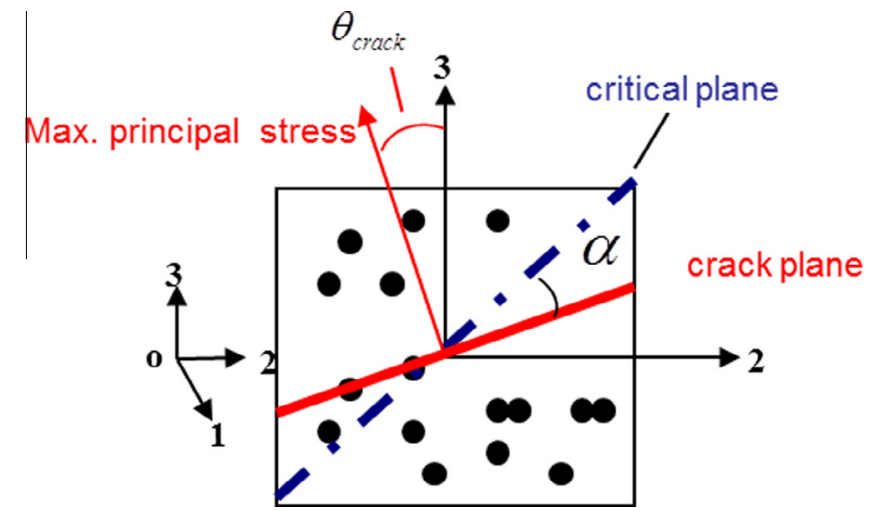

Fig. 11. Illustration of critical plane and crack plane in the material coordinate system. The angles between crack plane and axis $3\left(\boldsymbol{0}^{\circ}<\theta_{\text {crack }}<\mathbf{9 0}\right)$, and the angle between crack plane and critical plane $(\alpha)$ are depicted. refers to 'critical plane'). $f(N)$ and $t(N)$ are tension-tension and shear fatigue strengths at load cycle $N$, represented by the input $S-N$ curves of UD composite. $k$ and $\beta$ are material parameters which can be determined by uniaxial and torsional fatigue tests. Based on Eq. (9), a 3-D failure criterion that accounts for damage accumulation using Miner's rule was proposed [12].

\subsection{Anisotropic damage model}

The post-damage stiffness degradation is described by the anisotropic damage model [25] - Fig. 12. The coordinate system $1-2-3$ is the material coordinate system in an impregnated fibre bundle. Damage mode 1 delineates fibre rupture while the modes 2-4 are for inter-fibre cracks. For each of the damage modes there is a corresponding damage tensor.

$\boldsymbol{D}=\sum_{i} D_{i} \vec{n}_{i} \otimes \vec{n}_{i}=\left[\begin{array}{ccc}D_{1} & 0 & 0 \\ 0 & D_{2} & 0 \\ 0 & 0 & D_{3}\end{array}\right] \quad i=1,2,3$

where $D_{i}$ is the principle value and $\vec{n}_{i}$ is a unit vector. The physical meaning of the damage factor $D_{i}$ is the effective area reduction caused by cracks. In calculation, $D_{i}$ are zero for a virgin material and 0.99 for damage to avoid the numerical singularity. Using $D_{i}$, the degraded stiffness matrix in post-damage phase can be calculated as follows:

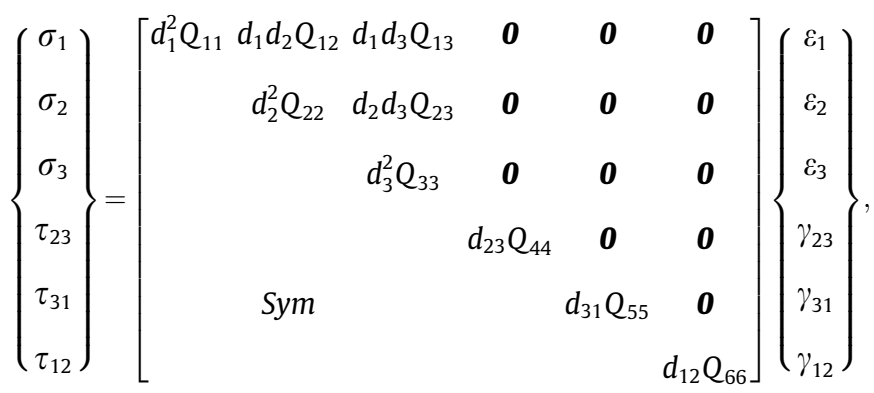

where $d$ and $D$ have the following relations,

\begin{tabular}{|c|c|c|c|c|c|}
\hline \multirow[b]{2}{*}{ Damage mode } & \multicolumn{4}{|c|}{ Anisotropic damage model for fiber bundle } & \multirow[b]{2}{*}{$\begin{array}{c}\text { Jsotropic } \\
\text { damage mode } \\
\text { for matrix }\end{array}$} \\
\hline & & Mode 2 & Mode 3 & & \\
\hline $\begin{array}{l}\text { Damage tensor } \\
{\left[\begin{array}{ccc}D_{1} & 0 & 0 \\
0 & D_{2} & 0 \\
0 & 0 & D_{3}\end{array}\right]}\end{array}$ & {$\left[\begin{array}{lll}1 & 0 & 0 \\
0 & 1 & 0 \\
0 & 0 & 1\end{array}\right]$} & {$\left[\begin{array}{lll}0 & 0 & 0 \\
0 & 1 & 0 \\
0 & 0 & 0\end{array}\right]$} & {$\left[\begin{array}{lll}0 & 0 & 0 \\
0 & 0 & 0 \\
0 & 0 & 1\end{array}\right]$} & {$\left[\begin{array}{lll}0 & 0 & 0 \\
0 & 1 & 0 \\
0 & 0 & 1\end{array}\right]$} & {$\left[\begin{array}{lll}1 & 0 & 0 \\
0 & 1 & 0 \\
0 & 0 & 1\end{array}\right]$} \\
\hline
\end{tabular}

Fig. 12. Damage modes and the corresponding damage tensor [26]. $D_{1}, D_{2}$ and $D_{3}$ are principle values of damage tensor, whose application is given in [1]. 


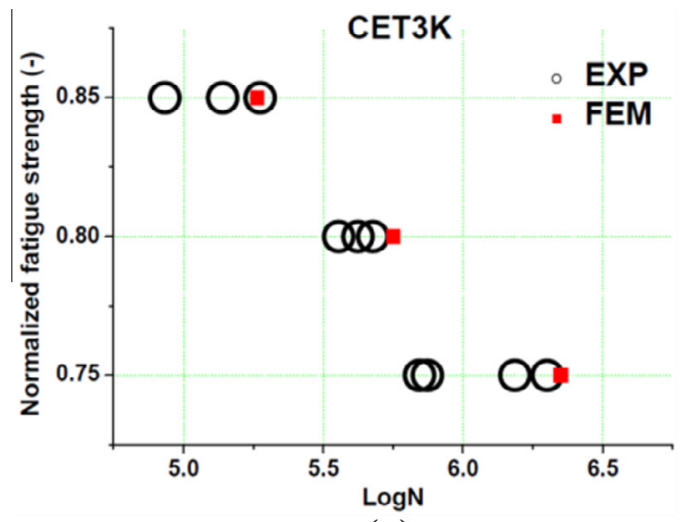

(a)

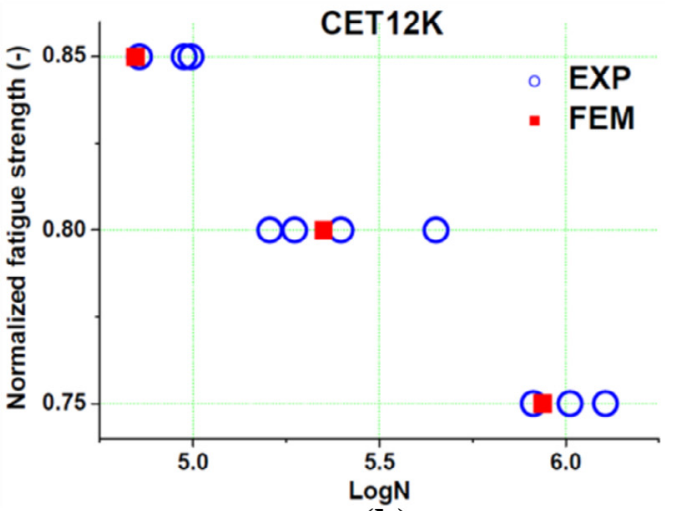

(b)

Fig. 13. Comparison of normalised $S-N$ plot: (a) CET3K and (b) CET12K.

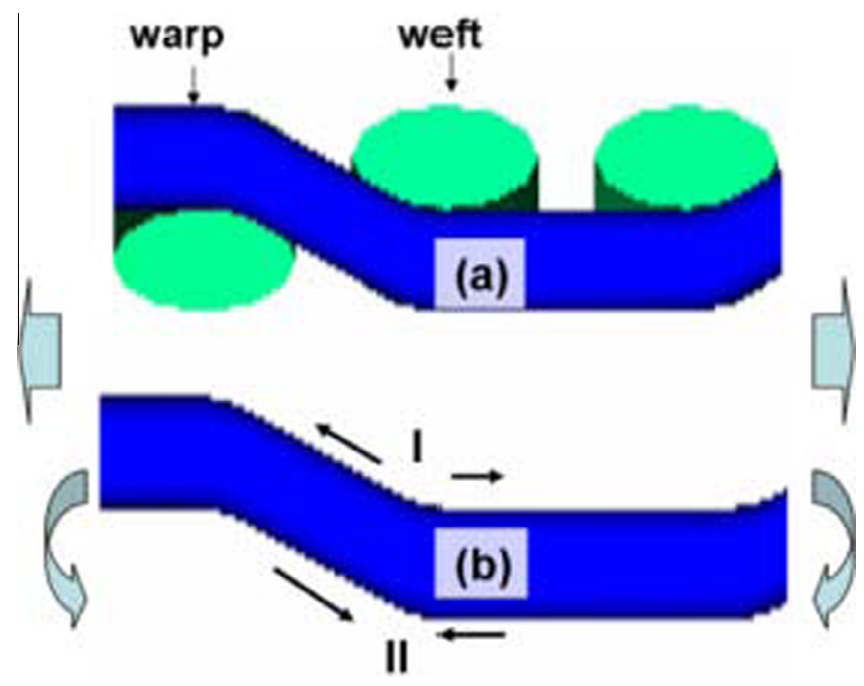

Fig. 14. Stress concentration introduced by crimp in warp tows: (a) a profile of impregnated twill weave preform in the composite; (b) bending effect and stress concentration at location I.
$\left\{\begin{array}{ccc}d_{1}=1-D_{1}, & d_{2}=1-D_{2}, & d_{3}=1-D_{3} \\ d_{23}=\left(\frac{2 d_{2} d_{3}}{d_{2}+d_{3}}\right)^{2}, & d_{31}=\left(\frac{2 d_{3} d_{1}}{d_{3}+d_{1}}\right)^{2}, & d_{12}=\left(\frac{2 d_{1} d_{2}}{d_{1}+d_{2}}\right)^{2}\end{array}\right.$

\section{Comparison and discussion}

\subsection{Comparison of $S-N$ plot}

The experimental moduli-life curves are used to determine the catastrophic failure of the materials. As shown in Fig. 4(b), the averaged moduli right before failure for CET3K and CET12K are $85 \%$ and $75 \%$ of the static moduli, respectively. The calculation will terminate once the fatigue moduli drop to the $85 \%$ and $75 \%$ for CET3K and CET12K, respectively.

Based on the moduli-life curves, the predicted normalised fatigue strengths of the two types of twill weave composites are presented in Fig. 13(a) and Fig. 13(b) (solid symbols) together with the experimental data (hollow symbols). In these figures, the fatigue strengths are compared at the maximum fatigue load level of $75 \%$, $80 \%$ and $85 \%$ of the static strength. Reasonably good agreements have been obtained for CET12K. For CET3K, the predicted fatigue strengths tend to be slightly higher than the test data. However,

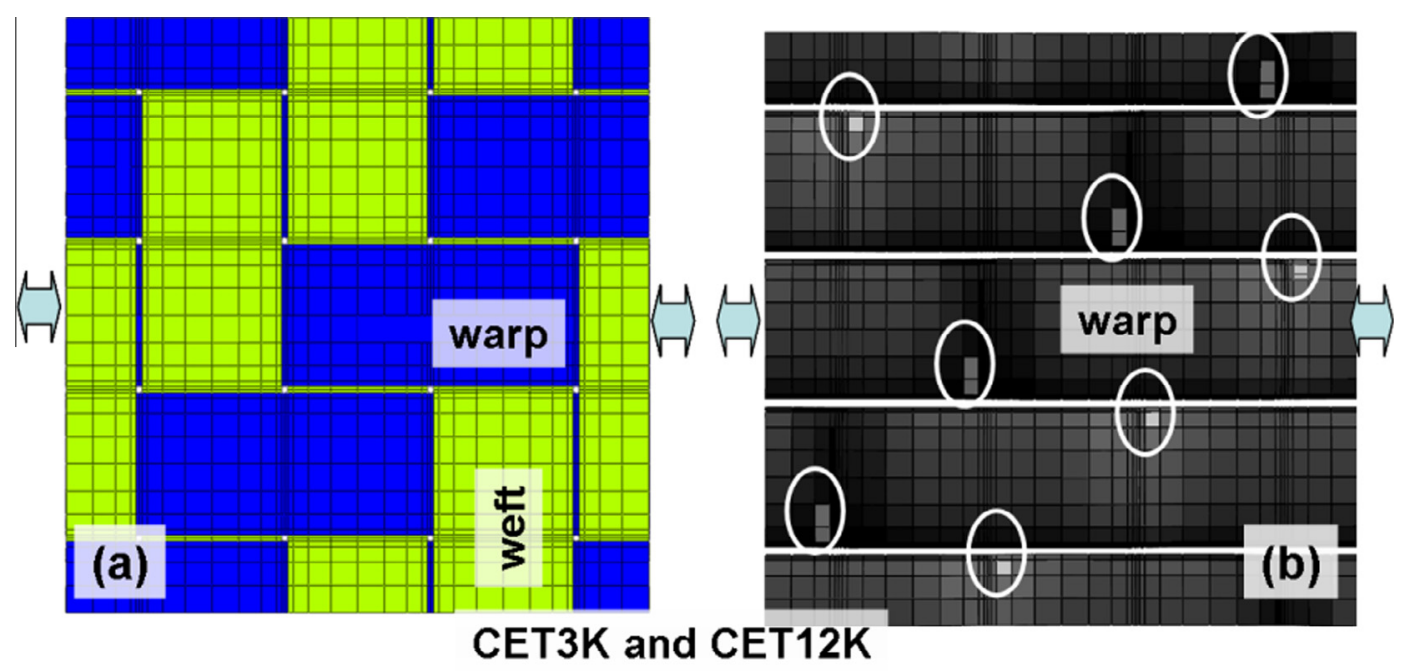

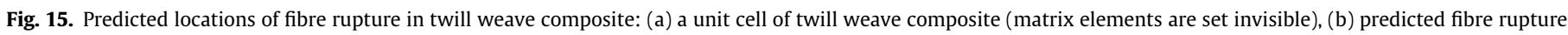
locations in the warp tows. 


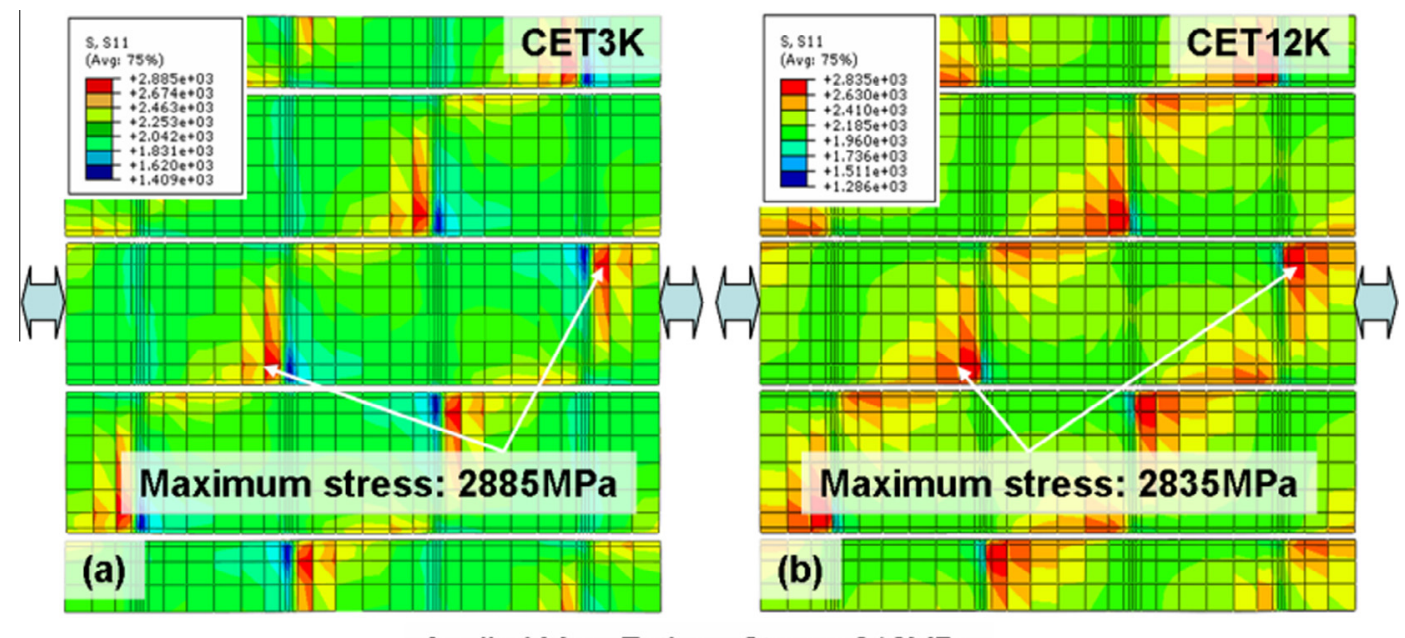

Applied Max. Fatigue Stress: 816MPa

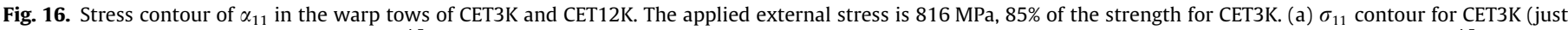

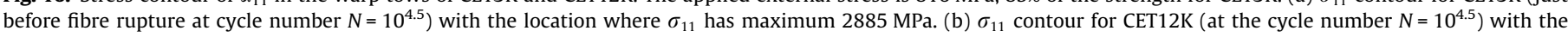
location where $\sigma_{11}$ has maximum $2835 \mathrm{MPa}$.

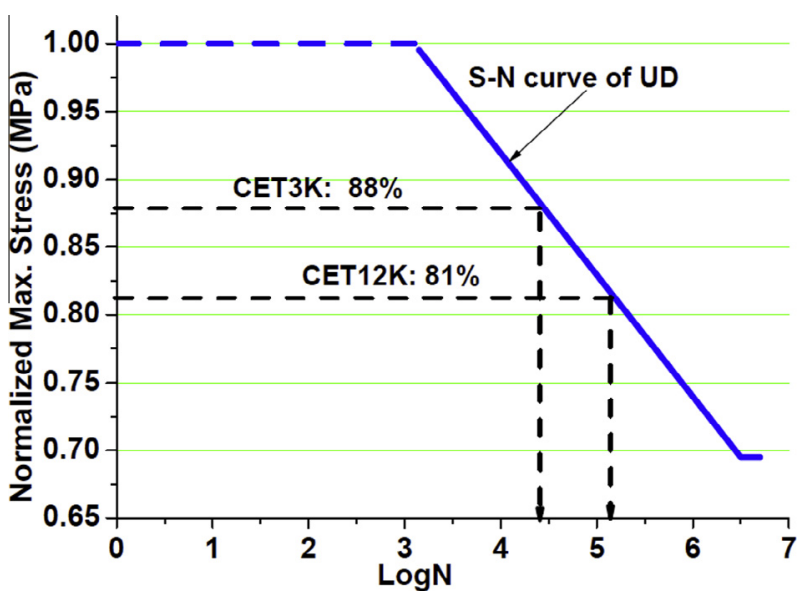

Fig. 17. Fatigue life estimation by using the normalised maximum fatigue stress and the $S-N$ curve of a UD composite (input data) before fibre rupture.

comparing to the large experimental scatters in high stress level fatigue tests, these deviations still fall into a reasonable range.

\subsection{Fibre rupture in warp tows}

Using the FE model, the locations of fibre rupture in the warp tow can be predicted. As shown in Fig. 15, CET3K and CET12K exhibit the same fibre rupture locations. The fibre rupture will initiate at the location where (1) warp tow and weft tow have a contact surface (in between the warp and weft tows at the cross points), and (2) the warp tow has the highest curvature [1] - Fig. 14(a). Fig. 14(b) shows the bending effect and where stress concentration takes place - location I.

In the following numerical tests, the same fatigue loadings of $816 \mathrm{MPa}, 85 \%$ of the static strength of CET3K, are applied to CET3K and CET12K, respectively. The predicted first fibre ruptures are at load cycle number $N=10^{4.5}$ and $N=10^{5.25}$ respectively for CET3K and CET12K. In Fig. 16 the $\sigma_{11}$ contours for CET3K and CET12K at cycle number $N=10^{4.5}$ are comparatively studied. Fig. $16(\mathrm{a})$ is the contour for CET3K, just before the fibre rupture. CET12K experiences the same fatigue loading and number of cycles without fibre rupture - Fig. 16(b). The maximum fatigue stress (stress concentration) in CET3K is $2885 \mathrm{MPa}, 50 \mathrm{MPa}$ higher than that in CET12K (2835 MPa).

The strengths of the impregnated tows can be calculated by using the Chamis' equations [27] and the tow $V_{F}$ for CET3K (67.2\%) and CET12K (71.4\%) - [1]. They are $3265 \mathrm{MPa}$ and $3500 \mathrm{MPa}$, respectively. Thus the normalised maximum stresses at the stress concentration locations for CET3K and CET12K in Fig. 16 are $88 \%$ and $81 \%$, respectively. The fatigue life can be roughly estimated by using the input $S-N$ curve shown in Fig. 2 (a). In Fig. 17, CET12K has lower normalised maximum fatigue stresses $(81 \%)$ at the stress concentration locations than CET3K (88\%) and hence postponed fibre ruptures $\left(10^{5.2}\right.$ cycles vs $10^{4.4}$ cycles) in the warp tows.

\section{Conclusion}

Two types of twill weave carbon fibre epoxy composite materials have been experimentally and numerically investigated under tension-tension fatigue loadings ( $85 \%, 80 \%$ and $75 \%$ of the static strength). They have the same fibre/resin system but different geometry of reinforcement (tow size and crimp). With the help of DIC, the elongation of the samples is calculated by subtracting the machine/grip deformation. Based on the sample elongation, the displacement $\mathrm{AI}$ and the fatigue moduli are obtained as well as the $S-N$ plots. From the experimental data, we conclude that:

1. CET12K has higher (18\% higher) fatigue strengths as well as static strength compared to CET3K.

2. The displacement AI after the fatigue tests for CET12K is averaged at $0.45 \mathrm{~mm}$, which is much higher than that of CET3K, $0.18 \mathrm{~mm}$ - Fig. 4 ; the modulus for CET12K immediately before catastrophic failure has decreased to $75 \%$ of the virgin modulus and this value for CET3K is $85 \%$ - Fig. 4(b). One reason could be the severe fatigue damage during the fatigue for CET12K Fig. 5.

3. The curves of displacement AI vs fatigue life are divided into two groups by the material types. They tend to be dependent on the material types but irrelative to the load level.

4. The model is able to predict the materials' fatigue lives and the fibre rupture locations that are introduced by stress concentration. Higher crimp in CET3K introduces higher stress concentration and leads to shorter fatigue lives. 
As a summary, CET12K shows much better fatigue resistance than CET3K due to the flatter tow and lower stress concentration in warp tows. These phenomena can be well explained using the proposed fatigue model [12]. Nevertheless, a model with delamination is necessary and more studies have to be carried out on stochastic breakage of the fibres in tows during fatigue.

\section{References}

[1] Xu J, Lomov SV, Verpoest I, Daggumati S, Van Paepegem W, Degrieck J, et al. A progressive damage model of textile composites on meso-scale using finite element method: static damage analysis. J Compos Mater 2013.

[2] Degrieck J, Van Paepegem W. Fatigue damage modeling of fibre-reinforced composite materials: review. Appl Mech Rev 2001;54:279-300.

[3] Post N, Case S, Lesko J. Modeling the variable amplitude fatigue of composite materials: a review and evaluation of the state of the art for spectrum loading. Int J Fatigue 2008;30:2064-86.

[4] Passipoularidis V, Brøndsted P. Fatigue evaluation algorithms: review: Danmarks Tekniske Universitet, Risø Nationallaboratoriet for Bæredygtig Energi; 2010.

[5] Xu J. Meso-scale finite element fatigue modelling of textile composite materials [Ph.D. thesis]. <https://lirias.kuleuven.be/handle/123456789/ 312878>. Leuven: KUL; 2011.

[6] Baere ID, Paepegem WV, Degrieck J. On the nonlinear evolution of the Poisson's ratio under quasi-static loading for a carbon fabric-reinforced thermoplastic. Part II: analytical explanation. Polym Test 2009;28:324-30.

[7] Khan MZS, Mouritz AP. Fatigue behaviour of stitched GRP laminates. Compos Sci Technol 1996:56:695-701.

[8] Carvelli V, Tomaselli VN, Lomov SV, Verpoest I, Witzel V, den Broucke BV. Fatigue and post-fatigue tensile behaviour of non-crimp stitched and unstitched carbon/epoxy composites. Compos Sci Technol 2010;70:2216-24.

[9] Carvelli V, Gramellini G, Lomov SV, Bogdanovich AE, Mungalov DD, Verpoest Fatigue behavior of non-crimp 3D orthogonal weave and multi-layer plain weave E-glass reinforced composites. Compos Sci Technol 2010;70:2068-76.

[10] Nishikawa Y, Okubo K, Fujii T, Kawabe K. Fatigue crack constraint in plainwoven CFRP using newly-developed spread tows. Int J Fatigue 2006;28:1248-53.

[11] Carvelli V, Lomov SV. Fatigue of textile composites. Woodhead Publishing; 2015.
[12] Xu J, Lomov SV, Verpoest I, Daggumati S, Van Paepegem W, Degrieck J. A progressive damage model of textile composites on meso-scale using finite element method: fatigue damage analysis. Comput Struct 2015;152:96-112.

[13] De Baere I, Paepegem WV, Degrieck J. Electrical resistance measurement for in situ monitoring of fatigue of carbon fabric composites. Int J Fatigue 2010;32:197-207.

[14] Hanaki S, Yamashita M, Uchida H, Zako M. On stochastic evaluation of S-N data based on fatigue strength distribution. Int J Fatigue 2010;32:605-9.

[15] Shokrieh MM, Lessard LB. Progressive fatigue damage modeling of composite materials, part II: material characterization and model verification. J Compos Mater 2000;34:1081-116.

[16] Jamison RD, Schulte K, Reifsnider KL, Stinchcomb WW. Characterization and analysis of damage mechanisms in tension-tension fatigue of graphite/epoxy laminates. Eff Defects Compos Mater 1984:21-55.

[17] Daggumati S. Concurrent modelling and experimental analysis of meso-scale strain field and damage in woven composites under static and fatigue tensile loading; 2011. p. 146-79.

[18] Van Paepegem W, Degrieck J. Fatigue degradation modelling of plain woven glass/epoxy composites. Compos A Appl Sci Manuf 2001;32:1433-41.

[19] Liu Y, Mahadevan S. Multiaxial high-cycle fatigue criterion and life prediction for metals. Int J Fatigue 2005;27:790-800.

[20] Liu Y, Mahadevan S. A unified multiaxial fatigue damage model for isotropic and anisotropic materials. Int J Fatigue 2007;29:347-59.

[21] Lomov SV, Gusakov AV, Huysmans G, Prodromou A, Verpoest I. Textile geometry preprocessor for meso-mechanical models of woven composites. Compos Sci Technol 2000;60:2083-95.

[22] Verpoest I, Lomov SV. Virtual textile composites software WiseTex: integration with micro-mechanical, permeability and structural analysis, Compos Sci Technol 2005;65:2563-74.

[23] Kurashiki T, Nakai H, Hirosawa S, Imura M, Zako M, Verpoest I, et al. Mechanical behaviours for textile composites by FEM based on damage mechanics. Key Eng Mater 2007;334-335:257-60.

[24] Lomov SV, Ivanov DS, Verpoest I, Zako M, Kurashiki T, Nakai H, et al. Meso-FE modelling of textile composites: road map, data flow and algorithms. Compos Sci Technol 2007;67:1870-91.

[25] Murakami S. Mechanical modeling of material damage. J Appl Mech 1988:55:280-6.

[26] Zako M, Uetsuji Y, Kurashiki T. Finite element analysis of damaged woven fabric composite materials. Compos Sci Technol 2002;63:507-16.

[27] Chamis CC. Mechanics of composite-materials - past, present, and future. ] Compos Technol Res 1989;11:3-14. 